\title{
Health Care Resource Utilization and Costs in First-Line Treatments for Patients with Metastatic Melanoma in the United States
}

\author{
Andrew J. Klink, PhD, MPH; Bartosz Chmielowski, MD, PhD; Bruce Feinberg, DO; \\ Sama Ahsan, MD; Damion Nero, PhD; and Frank Xiaoqing Liu, PhD
}

\begin{abstract}
BACKGROUND: The treatment landscape for patients with metastatic melanoma has changed dramatically with the introduction of novel therapies, such as targeted therapies and immunotherapies, in recent years. Health care resource utilization (HCRU) and cost data are needed to further evaluate these treatments in a value-based health care system.

OBJECTIVE: To examine HCRU and total cost of care among U.S. metastatic melanoma patients treated with first-line systemic therapies, including immunotherapies, targeted therapies, and chemotherapy.

METHODS: A retrospective observational study was conducted using a U.S. claims database. Adults with $\geq 2$ claims for melanoma and $\geq 1$ claim for metastasis between January 1, 2012, and June 30, 2017, were identified. Patients had pharmacy and medical enrollment $\geq 6$ months before and $\geq 3$ months following first-line treatment start. Per patient per month (PPPM) HCRU and costs were calculated by first-line treatment drug class: PD-1 inhibitors, CTLA-4 inhibitors, CTLA-4 + PD-1 combination, BRAF monotherapy, BRAF + MEK combination, and chemotherapy. Adjusted odds ratios (ORs) for HCRU were estimated by logistic regressions and adjusted costs were estimated by generalized linear models using log-link with gamma distribution to control for differences in patient characteristics across groups.
\end{abstract}

RESULTS: Among 1,599 metastatic melanoma patients (PD-1, $\mathrm{n}=255$; CTLA-4, $n=555$; CTLA-4 + PD-1, $n=88$; BRAF, $n=210$; BRAF + MEK, $\mathrm{n}=102$; chemotherapy $=389$ ), mean age ranged from $59-68$ years, and the majority were male $(62 \%)$. Any hospitalization during first-line treatment was less frequent among PD-1-treated patients (25.9\%) compared with $34.7 \%-45.5 \%$ of all other groups (all $P<0.05$ ). PPPM hospitalizations were lowest in PD-1 (0.06) compared with 0.09-0.16 across all other groups (all $P<0.05$ ), and PPPM emergency department (ED) visits were lowest in PD-1 (0.09) compared with 0.13-0.18 across all other groups (all $P<0.05$ ), except for BRAF+ MEK $(0.14, P=0.08)$. CTLA-4, CTLA-4 + PD-1, and BRAF + MEK had increased odds of hospitalization compared to PD-1 (adjusted ORs = 2.10, 2.35, 2.15, respectively; all $P<0.05)$. Total adjusted PPPM costs were significantly lower for PD-1 $(\$ 13,059)$ compared with CTLA-4 $(\$ 25,583)$, CTLA-4 + PD-1 (\$31,310), and BRAF + MEK $(\$ 21,517)$ and higher compared to $\operatorname{BRAF}(\$ 8,158)$ and chemotherapy $(\$ 6,361)$.

CONCLUSIONS: Hospitalizations and ED visits represent important HCRU for metastatic melanoma patients and were lowest among PD-1-treated patients compared with any other systemic therapies (except for ED visits when compared with BRAF+MEK). Total monthly costs varied substantially across first-line regimens and were significantly lower in PD-1-treated patients compared with patients treated with CTLA-4, CTLA-4+PD-1, and BRAF + MEK.

J Manag Care Spec Pharm. 2019;25(8):869-77

Copyright $\odot 2019$, Academy of Managed Care Pharmacy. All rights reserved.

\section{What is already known about this subject}

Substantial increase in effective therapies for patients with advanced melanoma in the past several years has been significantly improving patient outcomes.

There is a dearth of data on health care resource utilization (HCRU) and costs of newer agents to treat patients with metastatic melanoma, especially regarding PD-1 antibodies and immunotherapy combinations.

\section{What this study adds}

This retrospective observational study provides insights on HCRU and costs associated with contemporary systemic treatments, including immunotherapies and targeted therapies, for patients with metastatic melanoma in the United States.

Adjusted odds of hospitalization and emergency department visits across first-line therapies for metastatic melanoma were lowest among patients treated with PD-1 inhibitors.

Adjusted total monthly costs varied substantially across first-line therapies for metastatic melanoma and were significantly lower in PD-1-treated patients compared with patients treated with a CTLA-4 inhibitor, CTLA-4 + PD-1 combination, and BRAF/MEK combination.

$\mathrm{M}$ elanoma is the fifth most common cancer in the United States, representing 5.3\% of all new cancer cases. ${ }^{1}$ Over the past few years, several new treatment options have emerged for patients with unresectable or metastatic melanoma. ${ }^{2}$ Treatments now include monotherapy and combination and sequential therapies of checkpoint inhibitors and inhibitors targeting the mitogen-activated protein kinase (MAPK) pathway. Ipilimumab, an anti-cytotoxic T-lymphocyte antigen-4 (anti-CTLA-4), was the first checkpoint inhibitor approved by the U.S. Food and Drug Administration (FDA) in 2011; it was followed by FDA approvals of antiprogrammed cell death-1 (PD-1) antibodies pembrolizumab and nivolumab in 2014. Nivolumab in combination with ipilimumab was the first combination of checkpoint inhibitors approved by the FDA in 2015. Targeted therapies include the BRAF inhibitors vemurafenib, dabrafenib, and encorafenib, indicated for patients who test positive for the BRAF V600 mutations, and the MEK inhibitors trametinib, cobimetinib, and binimetinib, which are 
administered in combination with BRAF inhibitors. ${ }^{3}$ The use of these newer immunotherapies and targeted therapies has substantially improved overall survival among patients with metastatic melanoma but at greater economic impacts. ${ }^{4-15}$

Few economic studies exist that evaluate the effect of the newer immunotherapy and targeted therapy agents on the health care resource utilization (HCRU) and costs among patients treated for metastatic melanoma, particularly in a population of patients aged $>65$ years. ${ }^{2,5}$ Several studies exist that describe resource use and costs before the introduction of immunotherapy agents. ${ }^{2}$ Experience with ipilimumab monotherapy has been reported, although this has been limited to commercially insured populations and/or not limited to the first-line therapy setting. ${ }^{16,17}$ A recent analysis by Ghate et al. (2018), also conducted among commercially insured patients, assessed HCRU and found no difference in inpatient hospitalizations and emergency department (ED) visits between patients treated with first-line dabrafenib plus trametinib and patients treated with first-line nivolumab or pembrolizumab monotherapy (2.3 vs. 2.2 inpatient hospitalizations per patient-year, respectively; 1.6 vs. 2.1 ED visits per patient-year, respectively). ${ }^{18}$ While others have modeled U.S. costs among patients with metastatic melanoma treated with immunotherapies, few data exist on a limited set of immunotherapies. ${ }^{18-21}$ Very little information is available on metastatic melanoma patients treated with all currently available immunotherapies and targeted therapies.

Other existing economic studies evaluating specific subsets of patients and costs include the economic burden of metastatic melanoma patients with brain metastases, the economic burden associated with adverse events in patients with metastatic melanoma, and the economic burden in elderly metastatic and high-risk patients. ${ }^{22-24}$ Further, other studies have addressed the economic burden of metastatic melanoma in European health care settings, which differ greatly from that of the United States. ${ }^{25}$ However, the total burden of the disease has yet to be described in the current landscape of available systemic therapies.

Given the limitations of the existing literature characterizing the economic burden of metastatic melanoma in specific subgroups and in a period before the introduction of newer immunotherapy agents, this study provides timely data to understand the economic burden, in terms of HCRU and costs of the currently available first-line agents, including the most recently approved PD-1 inhibitors, in both commercial and government payer (Medicare and Medicaid) settings. The analyses that follow represent the first U.S. study to describe patients with metastatic melanoma treated with contemporary immunotherapies and targeted therapies, which include PD-1 inhibitors and CTLA-4 inhibitors, the combination of a CTLA-4 inhibitor and PD-1 inhibitor, targeted monotherapy (BRAF inhibitors), and targeted combination therapy (BRAF inhibitors plus MEK inhibitors), as well as with older agents involving traditional chemotherapy.

\section{Methods}

\section{Data Source}

This study was a retrospective, observational, cohort study of patients identified from Inovalon's Medical Outcomes and Research for Effectiveness and Economics $\left(\mathrm{MORE}^{2}\right)$ Registry. The MORE ${ }^{2}$ Registry is a payer database that compiles longitudinal claims data from over 100 U.S. health plans with 129 million unique covered patient lives since 2001, including more than 12 million cancer patients. The database contains adjudicated medical and pharmacy claims from small, medium, and large payers and represents the largest combined managed Medicare/Medicaid dataset in the industry. Approximately $45 \%$ of individuals within the dataset are covered by managed Medicare/Medicaid plans, with the remaining 55\% covered by nongovernmental, employer-sponsored plans. Data include patient demographic records, hospital discharge records, procedural records, practitioner profiles, eligibility and enrollment status, costs, and laboratory orders and results. Payers contributing data to the database update at least yearly, with approximately $40 \%$ updating quarterly. This study received exemption from Western Institutional Review Board because of the use of retrospective deidentified claims data.

\section{Study Population}

Patients were selected from the database if they had a melanoma diagnosis ( $\geq 2$ claims with International Classification of Diseases, Ninth/Tenth Revision, Clinical Modification [ICD9/10-CM] codes 172.x/C43.x) between January 1, 2012, and June 30, 2017, had a subsequent diagnosis of metastasis $(\geq 1$ claim with codes 196.x-198.x/C77.x-C79.x), had a systemic therapy for melanoma, and were aged $\geq 18$ years at metastatic melanoma diagnosis date. Patients on active treatment for a second cancer diagnosis, identified as participating in a clinical trial, or diagnosed with AIDS/HIV or a pregnancy during the study period were excluded.

The index date for each patient was set at the earliest date of first-line systemic therapy for melanoma; first-line therapy was identified by the earliest claim for systemic therapy following the diagnosis of melanoma. Patients included in the study cohort were required to have both pharmacy and medical enrollment with $\geq 6$ months of continuous medical and pharmacy enrollment before the index date and $\geq 3$ months of continuous medical and pharmacy enrollment in the follow-up period, which ended September 30, 2017. Data for each patient included claims until the last date of continuous enrollment or the end of the study period.

Patients were assigned to 1 of 6 treatment groups based on their first-line regimen identified from claims: anti-PD-1 monotherapy (PD-1, which included pembrolizumab or nivolumab), CTLA-4 inhibitor monotherapy (CTLA-4, ipilimumab), CTLA-4 inhibitor plus anti-PD-1 combination therapy (CTLA-4 + PD-1, which included ipilimumab plus nivolumab), 


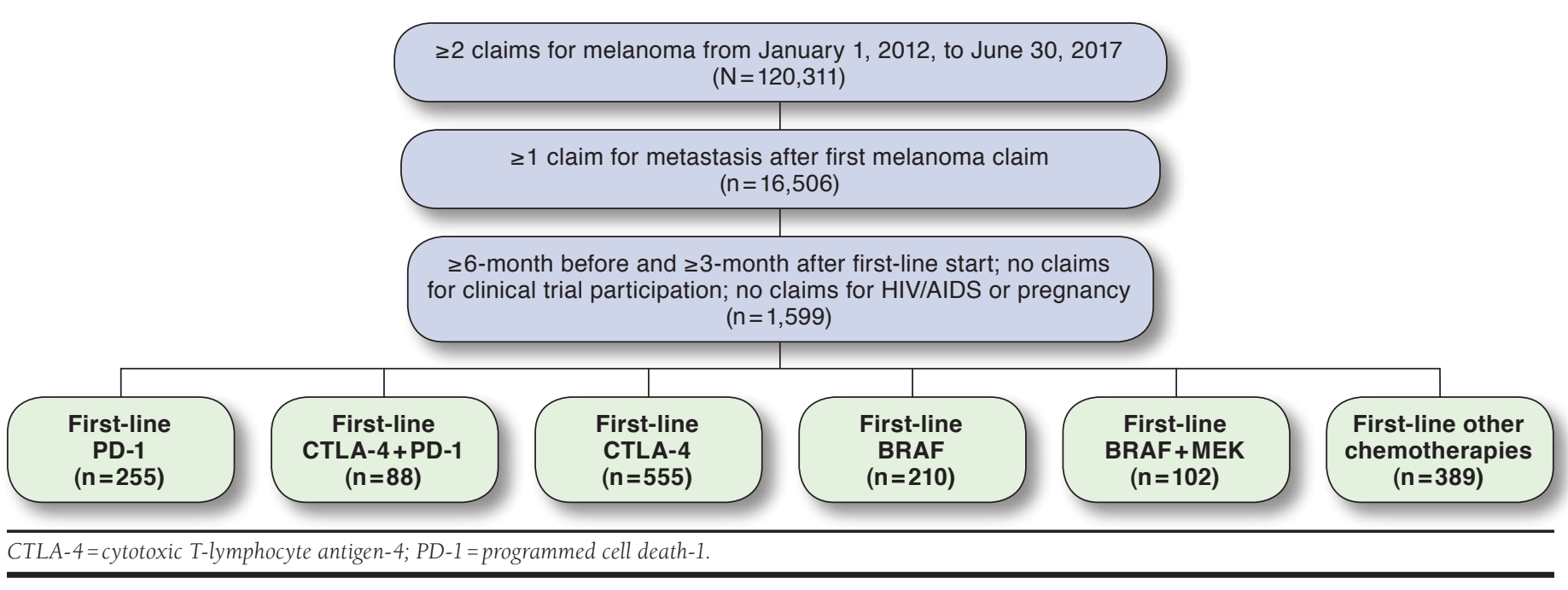

BRAF inhibitor monotherapy, BRAF plus MEK inhibitor combination therapy (BRAF+MEK), and other chemotherapies. Patients classified into the CTLA-4+PD-1 treatment group had to have claims for each within a day of each other and could have PD-1 monotherapy following the 16 weeks or less of combination therapy. BRAF inhibitors included vemurafenib and dabrafenib, and MEK inhibitors included trametinib and cobimetinib. Other chemotherapies included the remaining systemic chemotherapies for melanoma not elsewhere classified (e.g., paclitaxel, dacarbazine, temozolomide).

First-line therapy consisted of the time from the start of therapy until a change in regimen, end of continuous enrollment, or end of the study period, whichever occurred first. A gap in claims for the same regimen of $>45$ days ended the line of therapy, except for CTLA-4 monotherapy given the set number of treatment administrations (i.e., 4 doses over 16 weeks), where follow-up continued until a change in regimen, end of continuous enrollment, or end of the study period.

\section{Study Variables}

Patient characteristics (e.g., age at initiation of first-line therapy; sex; commercial insurance; Charlson Comorbidity Index [CCI] score at initiation of first-line therapy; and use of supportive care agents [antiemetics, growth factors, corticosteroids, and infliximab]) were summarized using descriptive statistics. ${ }^{26,27}$ HCRU was reported by all-cause resources during first-line treatment for the following categories: inpatient hospitalization; outpatient visits (composed of ED visits, outpatient office visits, radiation treatment, diagnostic radiology, laboratory services, and other outpatient care); melanoma treatment; and nonsystemic melanoma treatment pharmacy fills. Costs were inflated to 2017 U.S. dollars using the Consumer Price Index for medical care. ${ }^{28}$ Standardized costs were used in lieu of paid costs, since the latter were only available for a subset of patients in the study cohort. Standardized costs were derived from the Centers for Medicare \& Medicaid Services costs and were applied to observed HCRU for each patient during first-line therapy. ${ }^{29,30}$

\section{Statistical Analysis}

The number and proportion of patients with at least 1 of the resources of interest were calculated, as was the mean (median, standard deviation [SD]) number of the resources of interest among all patients and among patients with at least 1 claim for the resource of interest for each treatment group. HCRU was reported as per patient per month (PPPM) among all patients (whether they had the resource or not) to account for differences in follow-up time.

Comparisons of the proportion of patients with and rates (PPPM) of HCRU of interest across treatment cohorts, using PD-1 monotherapy as the referent, were made on univariate analyses by chi-square tests and t-tests to assess differences in categorical and continuous variables, respectively. To account for differences in baseline patient characteristics (age at initiation of first-line therapy, sex, commercial insurance, CCI score at initiation of first-line therapy, use of supportive care agents, U.S. region, and presence of brain metastases), multivariable logistic regressions were fit to estimate adjusted odds (odds ratios [ORs]) of an inpatient hospitalization and an ED visit during first-line therapy, given a patient's first-line treatment, using PD-1 as the referent. The list of characteristics for which estimates were adjusted were determined a priori, as deemed clinically relevant by clinicians on the study team. 
TABLE 1 Demographics and Patient Characteristics ( $N=1,599)$

\begin{tabular}{|c|c|c|c|c|c|c|c|c|c|c|c|c|c|c|c|c|c|}
\hline \multirow[b]{2}{*}{ Male, n (\%) } & \multicolumn{2}{|c|}{$\begin{array}{c}\text { PD-1 } \\
(\mathrm{n}=255)\end{array}$} & \multicolumn{2}{|c|}{$\begin{array}{l}\text { CTLA-4+PD-1 } \\
(\mathbf{n}=88)\end{array}$} & \multirow{2}{*}{\begin{tabular}{|c|}
$\begin{array}{c}P \\
\text { Value }^{\mathrm{a}}\end{array}$ \\
0.921 \\
\end{tabular}} & \multicolumn{2}{|c|}{$\begin{array}{l}\text { CTLA-4 } \\
(\mathrm{n}=555)\end{array}$} & \multirow{2}{*}{\begin{tabular}{|c|}
$\begin{array}{c}P \\
\text { Value }^{\mathrm{a}}\end{array}$ \\
0.210
\end{tabular}} & \multicolumn{2}{|c|}{$\begin{array}{c}\text { BRAF } \\
(\mathrm{n}=210)\end{array}$} & \multirow{2}{*}{\begin{tabular}{|c|}
$\begin{array}{c}P \\
\text { Value }^{\mathrm{a}}\end{array}$ \\
0.666 \\
\end{tabular}} & \multicolumn{2}{|c|}{$\begin{array}{c}\text { BRAF + MEK } \\
(\mathrm{n}=102)\end{array}$} & \multirow{2}{*}{\begin{tabular}{|c|}
$\begin{array}{c}P \\
\text { Value }^{\mathrm{a}}\end{array}$ \\
0.373 \\
\end{tabular}} & \multicolumn{2}{|c|}{$\begin{array}{c}\text { Other } \\
\begin{array}{c}\text { Chemotherapies } \\
(\mathbf{n}=389)\end{array} \\
\end{array}$} & \multirow{2}{*}{\begin{tabular}{|c|c}
$\begin{array}{c}\boldsymbol{P} \\
\text { Value }^{\mathrm{a}}\end{array}$ \\
0.329 \\
\end{tabular}} \\
\hline & 158 & $(62.0)$ & 54 & $(61.4)$ & & 369 & $(66.5)$ & & 126 & $(60.0)$ & & 58 & (56.9) & & 226 & $(58.1)$ & \\
\hline $\begin{array}{l}\text { Age at treatment initia- } \\
\text { tion in years, mean } \pm S D\end{array}$ & 67.6 & \pm 13.4 & 63.1 & \pm 13.1 & 0.007 & 63.1 & \pm 12.8 & $<0.001$ & 59.0 & \pm 13.6 & $<0.001$ & 59.3 & \pm 11.9 & $<0.001$ & 66.2 & \pm 12.2 & 0.191 \\
\hline Median & 68.0 & & 65.0 & & & 63.0 & & & 59.0 & & & 60.0 & & & 67.0 & & \\
\hline Region, n (\%) & & & & & 0.657 & & & 0.142 & & & 0.006 & & & 0.077 & & & 0.392 \\
\hline Northeast & 65 & $(25.5)$ & 17 & $(19.3)$ & & 123 & $(22.2)$ & & 28 & (13.3) & & 13 & (12.7) & & 85 & $(21.9)$ & \\
\hline South & 45 & $(17.6)$ & 14 & $(15.9)$ & & 119 & (21.4) & & 49 & (23.3) & & 20 & (19.6) & & 76 & $(19.5)$ & \\
\hline West & 97 & (38.0) & 40 & $(45.5)$ & & 186 & $(33.5)$ & & 75 & $(35.7)$ & & 42 & (41.2) & & 133 & (34.2) & \\
\hline Midwest & 47 & (18.4) & 17 & $(19.3)$ & & 127 & (22.9) & & 56 & $(26.7)$ & & 27 & (26.5) & & 92 & $(23.7)$ & \\
\hline Unknown & 1 & $(0.4)$ & 0 & $(0.0)$ & & 0 & $(0.0)$ & & 2 & (1.0) & & 0 & $(0.0)$ & & 3 & $(0.8)$ & \\
\hline Insurance status, n (\%) & & & & & 0.024 & & & $<0.001$ & & & $<0.001$ & & & $<0.001$ & & & 0.004 \\
\hline Commercial & 67 & $(26.3)$ & 33 & $(37.5)$ & & 262 & $(47.2)$ & & 126 & $(60.0)$ & & 57 & (55.9) & & 139 & $(35.7)$ & \\
\hline Medicare & 120 & $(47.1)$ & 36 & $(40.9)$ & & 188 & (33.9) & & 50 & $(23.8)$ & & 34 & (33.3) & & 187 & $(48.1)$ & \\
\hline Medicaid & 36 & $(14.1)$ & 16 & $(18.2)$ & & 71 & $(12.8)$ & & 25 & (11.9) & & 9 & $(8.8)$ & & 38 & $(9.8)$ & \\
\hline Unknown/other & 32 & $(12.5)$ & 3 & $(3.4)$ & & 34 & $(6.1)$ & & 9 & $(4.3)$ & & 2 & (2.0) & & 25 & $(6.4)$ & \\
\hline $\begin{array}{l}\mathrm{CCI} \text { at treatment } \\
\text { initiation, mean } \pm \mathrm{SD}\end{array}$ & 2.8 & \pm 1.7 & 2.9 & \pm 1.9 & 0.781 & 2.4 & \pm 1.7 & 0.006 & 2.2 & \pm 1.5 & $<0.001$ & 2.3 & \pm 1.5 & 0.014 & 2.6 & \pm 1.8 & 0.168 \\
\hline Median & 2.0 & & 2.5 & & & 2.0 & & & 1.5 & & & 2.0 & & & 2.0 & & \\
\hline $\begin{array}{l}\text { Metastasis in brain and } \\
\text { spinal cord, } \mathrm{n}(\%)\end{array}$ & 57 & $(22.4)$ & 27 & $(30.7)$ & 0.117 & 189 & $(34.1)$ & 0.001 & 88 & (41.9) & $<0.001$ & 49 & (48.0) & $<0.001$ & 83 & $(21.3)$ & 0.760 \\
\hline $\begin{array}{l}\text { Follow-up period from } \\
\text { first-line start to the end } \\
\text { of follow-up in months, } \\
\text { mean } \pm \text { SD }\end{array}$ & 10.3 & \pm 6.5 & 8.8 & \pm 5.1 & 0.032 & 14.5 & \pm 12.4 & $<0.001$ & 13.1 & \pm 12.9 & 0.005 & 11.5 & \pm 8.1 & 0.180 & 16.4 & \pm 13.9 & $<0.001$ \\
\hline Median & 9.1 & & 7.8 & & & 10.6 & & & 8.4 & & & 9.2 & & & 11.6 & & \\
\hline
\end{tabular}

aP value calculated by t-test/Wilcoxon or chi-square/Fisher's exact test comparing means or proportions to PD-1 as indicated.

$C C I=$ Charlson Comorbidity Index; CTLA-4=cytotoxic T-lymphocyte antigen-4; PD-1 = programmed cell death-1; SD =standard deviation

Costs were calculated by all-cause total health care costs and for each of the HCRU categories previously described during first-line therapy. Mean (median, SD) costs for each HCRU of interest were reported among all patients and among patients with at least 1 claim for the resource of interest. Cost analyses were performed in parallel for all-cause resource use and reported as PPPM costs. Adjusted PPPM costs were estimated by generalized linear models using log-link and a gamma distribution to account for differences in baseline characteristics (age at initiation of first-line therapy, sex, commercial insurance, CCI score at initiation of first-line therapy, use of supportive care agents, U.S. region, and presence of brain metastases).

\section{Results}

\section{Baseline Characteristics}

There were 6 first-line treatment groups categorized among 1,599 metastatic melanoma patients with $\geq 6$ months of continuous medical and pharmacy enrollment records before and $\geq 3$ months after first-line treatment start (Figure 1): PD-1 ( $\mathrm{n}=255)$, CTLA-4 + PD-1 ( $\mathrm{n}=88)$, CTLA-4 $(\mathrm{n}=555)$, BRAF $(n=210), B R A F+\operatorname{MEK}(n=102)$, and other chemotherapies $(n=389)$. The majority was male $(57 \%-62 \%)$ across treatment groups. On average, those treated with first-line PD-1 were older at treatment initiation (mean $=67.6$ years) compared with other first-line treatment groups (range $=59.0-63.1$, all $P<0.05)$, except for other chemotherapy, which had a similar mean age of 66.2 years old at metastatic diagnosis $(P=0.19$; Table 1).

There were regional differences between patients treated with first-line BRAF compared with first-line PD-1: BRAF had a greater proportion of patients in the Midwest $(26.7 \%$ vs. $18.4 \% ; P<0.01)$ and a lower proportion of patients in the Northeast $(13.3 \%$ vs. $25.5 \%$; $P<0.01)$ compared with PD-1. The proportion of commercially insured patients was lower among PD-1 patients (26.3\%) compared with other treatment groups (range $=37.5 \%-60.0 \%$; all $P<0.05$ ). The CCI score was higher among PD-1 patients (mean $C C I=2.8$ ) compared with other groups (mean CCI range $=2.2-2.4$; all $P<0.05$ ), except for CTLA-4+PD-1 and other chemotherapies, which had similar mean index scores (2.9 and 2.6, respectively). The incidence of brain metastases was more common among CTLA-4 (34.1\%), BRAF (41.9\%), and BRAF + MEK (48.0\%) compared with PD-1 
Health Care Resource Utilization and Costs in First-Line Treatments for Patients with Metastatic Melanoma in the United States

TABLE 2 Unadjusted Health Care Resource Utilization During First-Line

\begin{tabular}{|c|c|c|c|c|c|c|c|c|c|c|c|c|c|c|c|c|c|}
\hline \multirow[b]{2}{*}{ Hospitalizations, n (\%) } & \multicolumn{2}{|c|}{$\begin{array}{c}\text { PD-1 } \\
(\mathrm{n}=255)\end{array}$} & \multicolumn{2}{|c|}{$\begin{array}{l}\text { CTLA-4 + PD-1 } \\
\quad(n=88)\end{array}$} & \multirow{2}{*}{\begin{tabular}{|c}
$\begin{array}{c}P \\
\text { Value }^{\mathrm{a}}\end{array}$ \\
0.001 \\
\end{tabular}} & \multicolumn{2}{|c|}{$\begin{array}{l}\text { CTLA-4 } \\
(\mathrm{n}=555) \\
\end{array}$} & \multirow{2}{*}{$\begin{array}{c}\begin{array}{c}P \\
\text { Value }^{\mathrm{a}}\end{array} \\
<0.001 \\
\end{array}$} & \multicolumn{2}{|c|}{$\begin{array}{c}\text { BRAF } \\
(n=210)\end{array}$} & \multirow{2}{*}{$\begin{array}{c}\begin{array}{c}P \\
\text { Value }^{\mathrm{a}}\end{array} \\
0.009\end{array}$} & \multicolumn{2}{|c|}{$\begin{array}{c}\text { BRAF + MEK } \\
(\mathbf{n}=102)\end{array}$} & \multirow{2}{*}{\begin{tabular}{|c|c}
$\begin{array}{c}P \\
\text { Value }^{\mathrm{a}}\end{array}$ \\
0.005
\end{tabular}} & \multicolumn{2}{|c|}{\begin{tabular}{|c|}
$\begin{array}{c}\text { Other } \\
\text { Chemotherapies } \\
(\mathrm{n}=389)\end{array}$ \\
\end{tabular}} & \multirow{2}{*}{\begin{tabular}{|c|c}
$\begin{array}{c}P \\
\text { Value }^{\mathrm{a}}\end{array}$ \\
0.018 \\
\end{tabular}} \\
\hline & 66 & $(25.9)$ & 40 & $(45.5)$ & & 221 & $(39.8)$ & & 78 & $(37.1)$ & & 42 & $(41.2)$ & & 135 & $(34.7)$ & \\
\hline Mean $\mathrm{PPPM} \pm \mathrm{SD}$ & 0.06 & \pm 0.13 & 0.14 & \pm 0.23 & 0.001 & 0.10 & \pm 0.20 & $<0.001$ & 0.11 & \pm 0.20 & 0.003 & 0.16 & \pm 0.32 & 0.003 & 0.09 & \pm 0.21 & 0.007 \\
\hline $\begin{array}{l}\text { Hospitalization le } \\
\text { f stay in days, }{ }^{b} \\
P P P M \pm S D\end{array}$ & 2.42 & \pm 1.58 & 2.33 & \pm 1.47 & 749 & 4.13 & \pm 2 & 0.310 & 2.49 & 1.75 & 806 & 3.29 & 6 & 055 & 3.70 & 4 & 0.360 \\
\hline $\begin{array}{l}11-\mathrm{ca} \\
\mathrm{adn}\end{array}$ & 35 & (13.7) & 16 & $(18.2)$ & 0.311 & 106 & $(19.1)$ & 0.061 & 48 & $(22.9)$ & 0.011 & 22 & $(21.6)$ & 0.068 & 76 & $(19.5)$ & 0.056 \\
\hline ED visits, $n$ & 102 & $(40.0)$ & 44 & $(50.0)$ & 0.102 & 261 & $(47.0)$ & 0.062 & 91 & (43.3) & 0.468 & 47 & $(46.1)$ & 0.293 & 169 & $(43.4)$ & 0.387 \\
\hline Mean $\mathrm{PPPM} \pm \mathrm{SD}$ & 0.09 & \pm 0.18 & 0.18 & \pm 0.24 & 0.004 & 0.13 & \pm 0.22 & 0.014 & 0.13 & \pm 0.22 & 0.030 & 0.14 & \pm 0.23 & 0.083 & 0.15 & \pm 0.31 & 0.006 \\
\hline Clinic/office & 252 & (98.8) & 88 & $(100.0)$ & 0.573 & 555 & $(100.0)$ & 0.031 & 208 & (99.0) & 1.000 & 100 & $(98.0)$ & 0.627 & 385 & (99.0) & 1.000 \\
\hline Mean $\mathrm{PPPM} \pm \mathrm{SD}$ & 2.43 & \pm 1.42 & 2.55 & \pm 1.88 & 0.604 & 2.54 & \pm 1.89 & 0.360 & 2.40 & \pm 1.69 & 0.813 & 2.11 & \pm 1.32 & 0.051 & 2.90 & \pm 2.19 & 0.001 \\
\hline harmacy & 239 & $(93.7)$ & 79 & (89.8) & 0.219 & 526 & (94.8) & 0.545 & 200 & (95.2) & 0.480 & 99 & (97.1) & 0.205 & 365 & (93.8) & 0.957 \\
\hline Mean $\mathrm{PPPM} \pm \mathrm{SD}$ & 2.07 & \pm 1.55 & 2.21 & \pm 1.65 & 0.489 & 2.17 & \pm 1.61 & 0.401 & 2.25 & \pm 1.74 & 0.252 & 2.25 & \pm 1.61 & 0.331 & 2.32 & \pm 1.73 & 0.065 \\
\hline
\end{tabular}

aP value calculated by t-test/Wilcoxon or chi-square/Fisher's exact test comparing means or proportions to PD-1 as indicated.

bLength of stay among those hospitalized.

'Excluded claims for melanoma treatment.

CTLA-4=cytotoxic T-lymphocyte antigen-4; ED=emergency department; PD-1 = programmed cell death-1; PPPM=per patient per month; SD=standard deviation.

(22.4\%; all $P<0.05)$ and was similar to PD-1 in CTLA-4+PD-1 (30.7\%) and other chemotherapies (21.3\%; Table 1).

\section{Health Care Resource Utilization}

HCRU among patients with metastatic melanoma treated with a first-line PD-1-blocking antibody was compared with the other treatment groups of interest (Table 2). A significantly lower proportion of PD-1 patients had at least 1 hospitalization during first-line treatment (25.9\%) compared with $45.5 \%$, $39.8 \%, 37.1 \%, 41.2 \%$, and $34.7 \%$ of patients treated with CTLA-4+PD-1, CTLA-4, BRAF, BRAF+MEK, and other chemotherapy, respectively (all $P<0.05$; Table 2 ). The rate of hospitalizations PPPM was statistically significantly lower among those treated with PD-1 (mean PPPM $=0.06)$ compared with all other treatment groups (mean PPPM range $=0.09-0.14$; all $P<0.01$; Table 2).

While not statistically significantly different, $40.0 \%$ of PD-1 patients had an ED visit during first-line treatment, while $50.0 \%, 47.0 \%, 43.3 \%, 46.1 \%$, and $43.4 \%$ of patients treated with a CTLA-4 + PD-1, CTLA-4, BRAF, BRAF + MEK, and other chemotherapy, respectively, had at least 1 ED visit during firstline treatment (Table 2). PPPM ED visits were statistically significantly lower among PD-1 (mean PPPM $=0.09$ ) patients compared with all other treatments (mean PPPM range $=0.13-0.18$; all $P<0.05$ ), except for BRAF + MEK, which was similar (mean PPPM $=0.14 ; P=0.08$ ). Nearly all patients had office visits and pharmacy claims (Table 2).

After adjusting for differences in baseline patient characteristics, patients treated with CTLA-4+PD-1, CTLA-4, or $\mathrm{BRAF}+\mathrm{MEK}$ were more than 2 times as likely to have an inpatient hospitalization compared with PD-1 during first-line treatment (all $P<0.05$; Table 3). Patients treated with CTLA-4 had adjusted odds that were 1.44 (95\% confidence interval $[\mathrm{CI}]=1.02$ 2.04; $P=0.038$ ) times as high as PD-1 patients (Table 3).

\section{Total Cost of Care}

Overall mean PPPM health care costs during first-line treatment were $\$ 12,424, \$ 30,558, \$ 24,945, \$ 8,068, \$ 21,444$, and $\$ 6,084$ among patients treated with PD-1, CTLA-4+PD-1, CTLA-4, BRAF, BRAF+MEK, and other chemotherapies, respectively (Table 4). These unadjusted costs were all significantly different compared with PD-1: overall mean PPPM costs were higher for CTLA-4 + PD-1, CTLA-4, and BRAF + MEK and lower for BRAF and chemotherapy. Mean PPPM hospitalization costs were statistically significantly lower in PD-1 (mean PPPM $=\$ 371$ ) compared with all other treatment groups (mean PPPM range $=\$ 598-\$ 1,066$; all $P<0.05$ ).

Mean PPPM ED visit costs were statistically significantly lower for PD-1 (mean PPPM $=\$ 35$ ) compared with CTLA-4 (mean PPPM $=\$ 49$ ) and CTLA-4 + PD-1 (mean PPPM $=\$ 61$; both $P<0.05$ ) but similar for all other treatments (mean PPPM range $=\$ 44-\$ 56)$. Total monthly health care costs were driven by mean monthly costs for metastatic melanoma treatment $(\$ 8,567, \$ 26,125, \$ 21,156, \$ 5,343, \$ 16,145$, and $\$ 1,160)$ for patients treated with PD-1, CTLA-4+PD-1, CTLA-4, BRAF, $\mathrm{BRAF}+\mathrm{MEK}$, and other chemotherapies, respectively (Table 4).

Adjusted total PPPM costs (i.e., sum of melanoma treatment, medical costs, and pharmacy costs) were $\$ 13,059$, $\$ 25,583, \$ 8,158, \$ 21,517, \$ 6,361$, and $\$ 31,310$ for PD-1, CTLA-4 + PD-1, CTLA-4, BRAF, BRAF + MEK, and other chemotherapies. Compared with PD-1 patients, those treated with CTLA-4+PD-1, CTLA-4, BRAF, BRAF+MEK, and other 


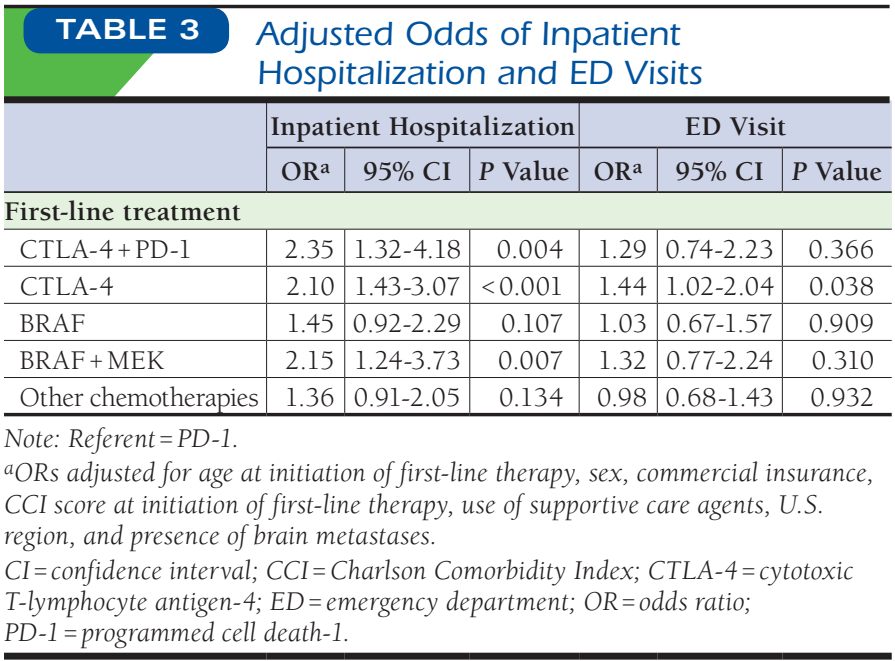

chemotherapies had incremental adjusted PPPM costs of $\$ 18,162, \$ 12,123,-\$ 4,706, \$ 8,273$, and $-\$ 6,049$, respectively (all $P<0.01 ;$ Table 4).

\section{Discussion}

In the last few years, multiple novel drugs, including immunotherapies, targeted therapies, and their combination therapies, received FDA approval for the treatment of patients with metastatic melanoma-all of which received a first-line indication. As our health care delivery transitions from a volume- to a value-based care design, real-world evidence, which includes the real-world cost of care, will be critical to guide payers and providers in optimal treatment and sequence selection. ${ }^{31,32}$ For those participating in innovative programs such as the Oncology Care Model, understanding respective treatment HCRU and cost is important to consider along with efficacy and toxicity.

There were important differences in HCRU among patients with metastatic melanoma treated with a first-line PD-1 inhibitor compared with the other treatment groups of interest, which may help health care decision makers differentiate first-line therapies. In particular, there were significantly fewer patients (25.9\%) treated with a PD-1-blocking antibody who had a hospitalization during first-line treatment, while 35\%-46\% of patients treated with another first-line regimen had at least 1 hospitalization during this stage of therapy. Rates of PPPM hospitalizations were lower among those treated with first-line PD-1 compared with all other treatment groups. Similarly, while not statistically significantly different, fewer patients (40\%) treated with a PD-1-blocking antibody had an ED visit during first-line therapy, while $43 \%-50 \%$ of patients treated with another first-line regimen had at least $1 \mathrm{ED}$ visit during this stage of therapy. Rates of PPPM ED visits were lower among PD-1 patients compared with all other treatments, except BRAF+MEK.
Overall mean adjusted PPPM health care costs during firstline treatment ranged from $\$ 6,361-\$ 31,310$ across all treatment groups analyzed and from $\$ 13,059-\$ 31,310$ among patients treated with an immunotherapy. These total monthly health care costs were driven by mean monthly costs for metastatic melanoma treatment, ranging from $\$ 1,160-\$ 26,125$ across all treatment groups analyzed and from $\$ 8,567-\$ 26,125$ among patients treated with an immunotherapy. Treatment costs of ipilimumab largely occurred in the first 12 weeks, given the fixed maximum duration of ipilimumab monotherapy, while costs for other metastatic melanoma treatments occurred over longer periods, influencing PPPM costs when costs were averaged across the duration of first-line therapy. While all patients were identified as having metastatic melanoma, disease severity within this group may explain some variation in health care costs. Further research is needed to elucidate drivers of higher health care costs in this population, which may include indicators of greater disease severity and/or poorer prognoses.

Comparisons of this study with previous studies are challenging or simply limited due to differences in study design and/or patient populations. However, Reyes et al. (2013) found a mean PPPM of $\$ 11,464$ for patients with 1-3 metastases and $\$ 15,662$ mean PPPM for patients with $\geq 4$ metastasis. ${ }^{33}$ They analyzed a younger patient population (mean age 57.2 years vs. 62 years in the current study) and derived data from a commercial payer database, which is likely not generalizable to the broader metastatic melanoma population across payer types where the mean age may be higher. Further, costs estimated by Reyes et al. were before the introduction of more costly melanoma treatments, including ipilimumab. Toy et al. (2015) estimated the mean total costs PPPM at $\$ 35,472$ among those treated with ipilimumab and $\$ 17,793$ for the vemurafenib cohort, albeit again among a commercially insured population. ${ }^{16}$

In the current study, we found a mean adjusted total cost PPPM for those treated with ipilimumab monotherapy to be similar $(\$ 31,310)$ to the Toy et al. results and a mean total cost PPPM for those treated with BRAF monotherapy (the current study included vemurafenib and dabrafenib, whereas Toy et al. included vemurafenib only; however, variation between these 2 is likely to be substantially lower $(\$ 8,158)$. Differences in the latter could be partially explained by the different time periods studied and the minimum follow-up period used ( 1 month vs. 3 months minimum in the Toy et al. and current studies, respectively).

Work by Chang et al. (2015) reports results of systemic therapies for metastatic melanoma before the introduction of nivolumab and pembrolizumab. Unadjusted monthly costs for vemurafenib were $\$ 17,223$ per patient and $\$ 65,313$ per patient for ipilimumab. ${ }^{17}$ It is important to point out that that analysis included any line of therapy, whereas the current study focused on the first-line setting. Further, the cohort analyzed by Chang et al. was limited to a commercially insured population with a 
TABLE 4 PPPM Health Care Costs During First-Line Treatment

\begin{tabular}{|c|c|c|c|c|c|c|c|c|c|c|c|}
\hline & & \multicolumn{2}{|l|}{$\begin{array}{c}\text { PD-1 } \\
(\mathrm{n}=255)\end{array}$} & $\begin{array}{l}\text { CTLA-4+PD-1 } \\
\quad(\mathrm{n}=88)\end{array}$ & Diff. $^{a}$ & $P$ Value $^{\mathrm{b}}$ & \multicolumn{2}{|c|}{$\begin{array}{l}\text { CTLA-4 } \\
(\mathrm{n}=555)\end{array}$} & \multicolumn{2}{|c|}{ Diff. $^{a}$} & $P$ Value ${ }^{b}$ \\
\hline \multicolumn{12}{|c|}{ Unadjusted all-cause PPPM cost in dollars, mean \pm SD } \\
\hline \multicolumn{2}{|l|}{ Total } & \multicolumn{2}{|c|}{$12,424 \pm 6,429$} & $30,558 \pm 21,368$ & 18,134 & $<0.001$ & \multicolumn{2}{|c|}{$24,945 \pm 17,408$} & \multicolumn{2}{|c|}{12,521} & $<0.001$ \\
\hline \multicolumn{2}{|l|}{ Melanoma treatment related } & \multicolumn{2}{|c|}{$8,567 \pm 4,212$} & $26,125 \pm 20,029$ & 17,558 & $<0.001$ & \multicolumn{2}{|c|}{$21,156 \pm 16,028$} & \multicolumn{2}{|c|}{12,589} & $<0.001$ \\
\hline \multicolumn{2}{|l|}{ Pharmacyc } & \multicolumn{2}{|c|}{$2,061 \pm 2,849$} & $2,336 \pm 3,063$ & 275 & 0.445 & \multicolumn{2}{|c|}{$1,958 \pm 2,473$} & \multicolumn{2}{|c|}{-103} & 0.619 \\
\hline \multicolumn{2}{|l|}{ Total medicald } & \multicolumn{2}{|c|}{$1,796 \pm 3,409$} & $2,097 \pm 1,951$ & 301 & 0.313 & \multicolumn{2}{|c|}{$1,831 \pm 2,594$} & & 35 & 0.884 \\
\hline Hospitalization & & $371 \pm 885$ & & $1,066 \pm 1,71$ & 695 & $<0.001$ & & $6 \pm 1,630$ & & 55 & $<0.001$ \\
\hline ED & & $35 \pm 75$ & & $61 \pm 93$ & 26 & 0.018 & & $9 \pm 124$ & & 14 & 0.044 \\
\hline Clinic/office visits & & $517 \pm 550$ & & $488 \pm 401$ & -28 & 0.604 & & $8 \pm 966$ & & 12 & 0.826 \\
\hline Radiation & & $48 \pm 199$ & & $204 \pm 512$ & 156 & 0.007 & & $1 \pm 294$ & & 23 & 0.197 \\
\hline Diagnostic radiology & & $108 \pm 172$ & & $92 \pm 131$ & -16 & 0.353 & & $0 \pm 149$ & & -8 & 0.503 \\
\hline Laboratory tests & & $60 \pm 68$ & & $63 \pm 63$ & 3 & 0.734 & & $6 \pm 100$ & & -4 & 0.520 \\
\hline Other outpatient visits & & $795 \pm 3,06$ & & $720 \pm 1,19$ & -75 & 0.745 & & $4 \pm 1,305$ & -1 & 71 & 0.392 \\
\hline $\begin{array}{l}\text { Adjustede all-cause total P } \\
\text { costs in dollars, mean ( } 95 \\
\end{array}$ & & $\begin{array}{c}13,059 \\
(11,263-15,14 \\
\end{array}$ & & $\begin{array}{c}31,310 \\
(25,890-37,86 \\
\end{array}$ & 18,162 & $<0.001$ & $(22,4$ & $\begin{array}{l}5,583 \\
5-29,199) \\
\end{array}$ & 12,1 & & $<0.001$ \\
\hline & & $\begin{array}{c}\text { BRAF } \\
(\mathbf{n}=210)\end{array}$ & Diff. $^{a}$ & $P$ Value $^{\mathrm{b}}$ & $\begin{array}{c}\text { BRAF + MEK } \\
(n=102)\end{array}$ & Diff. $^{a}$ & $P$ Value $^{\mathrm{b}}$ & $\begin{array}{r}\text { Oth } \\
\text { Chemoth } \\
(n=3\end{array}$ & ies & Diff. $^{a}$ & $P$ Value $^{\mathrm{b}}$ \\
\hline Unadjusted all-cause $P$ & $\cos$ & st in dollars, $n$ & $\mathrm{an} \pm \mathrm{SD}$ & & & & & & & & \\
\hline Total & & $8,068 \pm 4,907$ & $-4,356$ & $<0.001$ & $21,444 \pm 13,133$ & 9,020 & $<0.001$ & 6,084 & & $-6,340$ & $<0.001$ \\
\hline $\begin{array}{l}\text { Melanoma treatment } \\
\text { related }\end{array}$ & & $5,343 \pm 3,517$ & $-3,224$ & $<0.001$ & $16,145 \pm 11,597$ & 7,578 & $<0.001$ & $1,160 \pm$ & & $-7,407$ & $<0.001$ \\
\hline Pharmacyc & & $1,711 \pm 2,313$ & -351 & 0.144 & $3,534 \pm 5,168$ & 1,472 & 0.008 & $2,307 \pm$ & 905 & 246 & 0.357 \\
\hline Total medicald & & $1,014 \pm 1,317$ & -782 & 0.001 & $1,766 \pm 2,189$ & -30 & 0.922 & 2,617 & 956 & 822 & 0.005 \\
\hline Hospitalization & & $598 \pm 1,288$ & 227 & 0.031 & $1,047 \pm 2,016$ & 676 & 0.001 & $638 \pm$ & 728 & 267 & 0.010 \\
\hline ED & & $44 \pm 112$ & 9 & 0.316 & $56 \pm 124$ & 22 & 0.100 & $44 \pm$ & & 9 & 0.212 \\
\hline Clinic/office visits & & $312 \pm 452$ & -205 & $<0.001$ & $424 \pm 386$ & -93 & 0.073 & $1,079=$ & 336 & 562 & $<0.001$ \\
\hline Radiation & & $21 \pm 97$ & -27 & 0.057 & $189 \pm 1,202$ & 141 & 0.241 & $55 \pm$ & & 7 & 0.716 \\
\hline Diagnostic radiology & & $68 \pm 163$ & -40 & 0.011 & $113 \pm 179$ & 5 & 0.809 & $81 \pm$ & & -28 & 0.037 \\
\hline Laboratory tests & & $26 \pm 38$ & -35 & $<0.001$ & $28 \pm 37$ & -32 & $<0.001$ & $29 \pm$ & & -32 & $<0.001$ \\
\hline Other outpatient visits & & $267 \pm 504$ & -528 & 0.007 & $513 \pm 769$ & -282 & 0.172 & $986 \pm$ & & 191 & 0.397 \\
\hline $\begin{array}{l}\text { Adjustede all-cause total } \\
\text { PPPM costs in dollars, } \\
\text { mean }(95 \% \text { CI }) \\
\end{array}$ & 8,15 & $58(7,024-9,473)$ & $-4,706$ & $<0.001$ & $\begin{array}{c}21,517(17,953- \\
25,791)\end{array}$ & 8,273 & $<0.001$ & $6,361(5,56$ & $7,278)$ & $-6,049$ & $<0.001$ \\
\hline 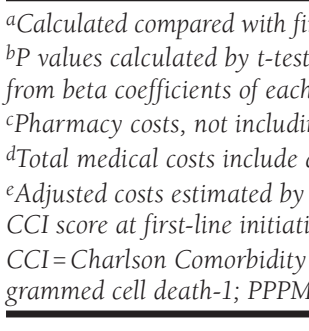 & $\begin{array}{l}g \text { cost } \\
l l \text { med } \\
\text { general } \\
\text { on; use }\end{array}$ & $\begin{array}{l}\text { PD-1 monotherap } \\
\text { paring means of ea } \\
\text { ment group compar } \\
\text { t of melanoma trea } \\
\text { dical claims (excluc } \\
\text { alized linear model } \\
\text { e of supportive car } \\
\text {; I = confidence in } \\
\text { patient per month; }\end{array}$ & $\begin{array}{l}\text { (for adju } \\
\text { ih treatme } \\
\text { ed with fir } \\
\text { ment (i.e., } \\
\text { ing pharm } \\
\text { using a ga } \\
\text { agents (a } \\
\text { erval; CT } \\
\text { SD = stanc }\end{array}$ & $\begin{array}{l}\text { Usted costs, diff } \\
\text { zent group com } \\
\text { irst-line PD-1 } \\
\text { e., BRAF/MEK } \\
\text { macy claims a } \\
\text { zamma distribu } \\
\text { (antiemetics, gr } \\
\text { TLA-4 = cytoto } \\
\text { ndard deviatio }\end{array}$ & $\begin{array}{l}\text { rence represents th } \\
\text { ared with first-line } \\
\text { lonotherapy). } \\
d \text { cost of melanom } \\
\text { ion and log-link to } \\
\text { wth factors, cortic } \\
\text { ic T-lymphocyte a }\end{array}$ & $\begin{array}{l}\text { emental } \\
\text { monotl } \\
\text { tment). } \\
\text { t for age } \\
\text { ids, or it } \\
-4 \text {; Diff. }\end{array}$ & $\begin{array}{l}\text { first-line } \\
\text { ximab); } \\
\text { ifference }\end{array}$ & $\begin{array}{l} \\
\text { tiation; } \\
\text { region; } \\
=\text { emerg }\end{array}$ & $\begin{array}{l}\text { me } \\
\text { sen }\end{array}$ & $\begin{array}{l}\text { insuran } \\
\text { brain } m\end{array}$ & $\begin{array}{l}\text { notherapy). } \\
\text { sts derived } \\
\text { nce status; } \\
\text { netastases. } \\
1=\text { pro- }\end{array}$ \\
\hline
\end{tabular}

mean age of 57.5 years; the current study describes mean ages of 63-68 years across first-line treatment groups.

Outside the United States, hospitalizations for ipilimumab have been reported among $57 \%$ of melanoma patients, which was higher than the $40 \%$ reported herein. ${ }^{34}$ Similar to the Chang et al. study, hospitalizations were considered in any line of therapy in which ipilimumab was given, whereas the current study focused on the first-line setting. Another study reported annual costs of hospitalizations in France among patients with melanoma before progression at 5,036 euros (2017), which, in current 2019 U.S. dollars, is roughly $\$ 5,720 .^{35}$ This estimate falls within the range of annualized PPPM costs associated with hospitalizations reported in this study across first-line treatment groups $(\$ 4,452-\$ 12,798)$. Additional studies in the United States assessing costs of care for metastatic melanoma that consider contemporary, recently approved systemic therapies are needed to make further comparisons to the results of the current study.

There are several important strengths of this current study. There is a dearth of data on the economic burden of 
metastatic melanoma in the context of the newer PD-1 inhibitors in commercial and government payer settings. Further, all FDA-approved therapies for metastatic melanoma at the time of this study were included in the analysis. This study begins to fill the economic evidence gap by using medical and pharmacy claims data for patients with metastatic melanoma outside of the clinical trial context. While this often presents methodological challenges, it depicts real use of medications among a population that is likely more diverse than that observed in the clinical trial setting. Differential follow-up time was partially addressed by the use of PPPM units, and odds of hospitalization and ED visits, as well as adjusted costs, were estimated by multivariable analyses to control for potential confounding due to differences in baseline patient characteristics. Results of this study may help quantify the economic burden of treatment for metastatic melanoma and help identify approaches that are less costly in this population.

\section{Limitations}

Limitations of this study should be considered to put the results presented into context. The clinical outcome of survival was not available from the dataset, which is an important factor when evaluating available treatments. Adjusted estimates in these analyses controlled for potential confounding, but inherent with any observational study, residual confounding or unobserved confounding may still be present. Standardized costs may differ from paid costs in the real world, since they remove any effect of specific payer reimbursement policies.

While payer claims data do provide valuable insight into real-world use and costs of treatments, they are collected for administrative purposes and not for research. Inherent with all payer claims-based analyses, the identification of metastatic melanoma relies on the accuracy of claims coding; errors of omission or commission are possible. While the follow-up time was up to 3 years following the launch of some of the newer agents, this relatively limited follow-up may result in some right censoring of patients. The costs and resource utilization presented in the current analyses represent those during the period immediately following the index date and may not represent the long-term clinical practice for patients with metastatic melanoma.

Further research is needed that builds upon the current study to characterize the clinical outcomes in this population treated with the newer immunotherapies, as well as with more recently approved regimens (e.g., encorafenib and binimetinib).
Future work should assess HCRU and costs of individual regimens and within-class regimens. With a better understanding of clinical outcomes in the real-world setting, a more complete representation of the overall burden of metastatic melanoma can be realized, ultimately leading to identification of appropriate therapies to treat this population.

\section{Conclusions}

Not long ago, the treatment landscape of melanoma drastically changed with the introduction of immunotherapies and the BRAF and MEK inhibitors. Now, the landscape has once again evolved to include the PD-1 inhibitors pembrolizumab and nivolumab in monotherapy, as well as the immunotherapy combination of ipilimumab plus nivolumab. This study provides an early and contemporary investigation into the use of PD-1 monotherapy, CTLA-4 monotherapy, CTLA-4+PD-1 combination therapy, BRAF inhibitor monotherapy, BRAF+MEK inhibitor combination therapy, and chemotherapy in the realworld setting among patients with metastatic melanoma in the United States.

Hospitalizations and ED visits represent important health care resources for patients with metastatic melanoma, and PPPM rates were lowest among PD-1 compared with any other systemic therapies (except for ED visits compared with BRAF+MEK). Total monthly costs varied substantially across first-line regimens and were significantly lower in PD-1 compared with CTLA-4, CTLA-4+PD-1, and BRAF+MEK. Differences in resource use and costs should be considered by health care decision makers in view of a value-based care framework, where cost is weighed alongside clinical effectiveness to inform the selection of the optimal first-line therapy for patients.

\section{Authors}

ANDREW J. KLINK, PhD, MPH; BRUCE FEINBERG, DO; and DAMION NERO, PhD, Cardinal Health Specialty Solutions, Columbus, Ohio. BARTOSZ CHMIELOWSKI, MD, PhD, Division of Hematology-Medical Oncology, University of California, Los Angeles. SAMA AHSAN, MD, and FRANK XIAOQING LIU, PhD, Merck E Co., Kenilworth, New Jersey.

AUTHOR CORRESPONDENCE: Frank Xiaoqing Liu, PhD, Merck \& Co, 2000 Galloping Hill Rd., Kenilworth, NJ 07033. Tel.: 267.305.1265; E-mail: xiaoqing.liu@merck.com. 


\section{DISCLOSURES}

This study was funded by Merck Sharp \& Dohme, a subsidiary of Merck \& Co. Klink, Feinberg, and Nero are employees of Cardinal Health Specialty Solutions, which received funding from Merck to conduct this study. Chmielsowki is a consultant to Merck but received no funding for the development of this manuscript. Ahsan and Liu are employees of Merck. Chmielowski reports advisory board/speaker fees from Bristol-Myers Squibb, Merck, Genentech/Roche, Iovance Biotherapeutics, HUYA Bioscience International, Compugen, Array BioPharma, Regeneron, Biothera, Janssen, and Novartis. Ahsan has a patent (US20160008380Al) pending.

\section{REFERENCES}

1. National Cancer Institute. SEER stat fact sheets: melanoma of the skin. Available at: https://seer.cancer.gov/statfacts/html/melan.html. Accessed March 17, 2019.

2. Whitman ED, Liu FX, Cao X, Diede SJ, Haiderali A, Abernethy AP Treatment patterns and outcomes for patients with advanced melanoma in US oncology clinical practices. Future Oncol. 2019;15(5):459-71.

3. National Comprehensive Cancer Network. NCCN Clinical Practice Guidelines in Oncology (NCCN Guidelines)-cutaneous melanoma version 1.2019. Available at: https://www.nccn.org/professionals/physician_gls/ recently_updated.aspx. Accessed March 17, 2019.

4. Ascierto PA, Del Vecchio M, Robert C, et al. Ipilimumab $10 \mathrm{mg} / \mathrm{kg}$ versus ipilimumab $3 \mathrm{mg} / \mathrm{kg}$ in patients with unresectable or metastatic melanoma: a randomised, double-blind, multicentre, phase 3 trial. Lancet Oncol. 2017;18(5):611-22

5. Ascierto PA, McArthur GA, Dreno B, et al. Cobimetinib combined with vemurafenib in advanced BRAF(V600)-mutant melanoma (coBRIM): updated efficacy results from a randomised, double-blind, phase 3 trial. Lancet Oncol. 2016;17(9):1248-60.

6. Dummer R, Ascierto PA, Gogas H, et al. Overall survival in COLUMBUS a phase 3 trial of encorafenib (ENCO) plus binimetinib (BINI) vs. vemurafenib (VEM) or enco in BRAF-mutant melanoma. J Clin Oncol. 2018;36 (15 Suppl):9504.

7. Long GV, Hauschild A, Santinami M, et al. Adjuvant dabrafenib plus trametinib in stage III BRAF-mutated melanoma. N Engl J Med. 2017;377 (19):1813-23

8. McArthur GA Chapman PB, Robert C et al. Safety and efficacy of vemurafenib in BRAF(V600E) and BRAF(V600K) mutation-positive melanoma (BRIM-3): extended follow-up of a phase 3, randomised, open-label study. Lancet Oncol. 2014;15(3):323-32

9. Petrella TM, Robert C, Richtig E, et al. Patient-reported outcomes in KEYNOTE-006, a randomised study of pembrolizumab versus ipilimumab in patients with advanced melanoma. Eur J Cancer. 2017;86:115-24.

10. Revicki DA, van den Eertwegh AJ, Lorigan P, et al. Health related quality of life outcomes for unresectable stage III or IV melanoma patients receiving ipilimumab treatment. Health Qual Life Outcomes. 2012;10:66.

11. Robert C, Long GV, Brady B, et al. Nivolumab in previously untreated melanoma without BRAF mutation. N Engl J Med. 2015;372(4):320-30.

12. Robert C, Schachter J, Long GV, et al. Pembrolizumab versus ipilimumab in advanced melanoma. N Engl J Med. 2015;372(26):2521-32.

13. Robert C, Schadendorf D, Messina M, Hodi FS, O'Day S; MDX010-20 investigators. Efficacy and safety of retreatment with ipilimumab in patients with pretreated advanced melanoma who progressed after initially achieving disease control. Clin Cancer Res. 2013;19(8):2232-39.

14. Schadendorf D, Larkin J, Wolchok J, et al. Health-related quality of life results from the phase III CheckMate 067 study. Eur J Cancer. 2017:82:80-91.

15. Wolchok JD, Chiarion-Sileni V, Gonzalez R, et al. Overall survival with combined nivolumab and ipilimumab in advanced melanoma. N Engl J Med. 2017;377(14):1345-56

16. Toy EL, Vekeman F, Lewis MC, Oglesby AK, Duh MS. Costs, resource utilization, and treatment patterns for patients with metastatic melanoma in a commercially insured setting. Curr Med Res Opin. 2015;31(8):1561-72.
17. Chang CL, Schabert VF, Munakata J, et al. Comparative health care costs in patients with metastatic melanoma in the USA. Melanoma Res. 2015;25(4):312-20.

18. Ghate SR, Ionescu-Ittu R, Burne R, et al. Healthcare resource utilization in patients with metastatic melanoma receiving first-line therapy with dabrafenib + trametinib versus nivolumab or pembrolizumab monotherapy. Curr Med Res Opin. 2018;34(12):2169-76.

19. Kohn CG, Zeichner SB, Chen Q, Montero AJ, Goldstein DA, Flowers CR. Cost-effectiveness of immune checkpoint inhibition in BRAF wild-type advanced melanoma. J Clin Oncol. 2017;35(11):1194-202.

20. Oh A, Tran DM, McDowell LC, et al. Cost-effectiveness of nivolumabipilimumab combination therapy compared with monotherapy for first-line treatment of metastatic melanoma in the United States. J Manag Care Spec Pharm. 2017;23(6):653-64. Available at: https://www.jmcp.org/doi/10.18553/ jmcp.2017.23.6.653

21. Wang J, Chmielowski B, Pellissier J, Xu R, Stevinson K, Liu FX. Costeffectiveness of pembrolizumab versus ipilimumab in ipilimumab-naive patients with advanced melanoma in the United States. J Manag Care Spec Pharm. 2017;23(2):184-94. Available at: https://www.jmcp.org/doi/ full/10.18553/jmcp.2017.23.2.184

22. Davis KL, Mitra D, Kotapati S, Ibrahim R, Wolchok JD. Direct economic burden of high-risk and metastatic melanoma in the elderly: evidence from the SEER-Medicare linked database. Appl Health Econ Health Policy. 2009;7(1):31-41.

23. Vekeman F, Cloutier M, Yermakov S, Amonkar MM, Arondekar B Duh MS. Economic burden of brain metastases among patients with metastatic melanoma in a USA managed care population. Melanoma Res. 2014;24(6):602-10.

24. Arondekar B, Curkendall S, Monberg M, et al. Economic burden associated with adverse events in patients with metastatic melanoma. J Manag Care Spec Pharm. 2015;21(2):158-64. Available at: https://www.jmcp.org/doi/ abs/10.18553/jmcp.2015.21.2.158.

25. Wehler E, Zhao Z, Pinar Bilir S, Munakata J, Barber B. Economic burden of toxicities associated with treating metastatic melanoma in eight countries. Eur J Health Econ. 2017;18(1):49-58.

26. Charlson ME, Pompei P, Ales KL, MacKenzie CR. A new method of classifying prognostic comorbidity in longitudinal studies: development and validation. J Chronic Dis. 1987;40(5):373-83.

27. Sundararajan V, Henderson T, Perry C, Muggivan A, Quan H, Ghali WA. New ICD-10 version of the Charlson Comorbidity Index predicted in-hospital mortality. J Clin Epidemiol. 2004;57(12):1288-94.

28. Health Resources \& Services Administration. Consumer Price Index for Medical Care (CPI). Available at: https://www.hrsa.gov/get-health-care/ affordable/hill-burton/cpi.html. Accessed March 19, 2019.

29. O'Donnell BE, Schneider KM, Brooks JM, et al. Standardizing Medicare payment information to support examining geographic variation in costs. Medicare Medicaid Res Rev. 2013;3(3)

30. Schousboe JT, Paudel ML, Taylor BC, et al. Estimating true resource costs of outpatient care for Medicare beneficiaries: standardized costs versus Medicare payments and charges. Health Serv Res. 2016;51(1):205-19.

31. Chee TT, Ryan AM, Wasfy JH, Borden WB. Current state of value-based purchasing programs. Circulation. 2016;133(22):2197-205.

32. Ryan AM, Rodgers PE. Linking quality and spending to measure value for people with serious illness. J Palliat Med. 2018;21(S2):S74-S80.

33. Reyes C, DaCosta Byfield S, Linke R, Satram-Hoang S, Teitelbaum AH. The burden of metastatic melanoma: treatment patterns, healthcare use (utilization), and costs. Melanoma Res. 2013;23(2):159-66.

34. McArthur GA, Mohr P, Ascierto PA, et al. Health care resource utilization and associated costs among metastatic cutaneous melanoma patients treated with ipilimumab (INTUITION study). Oncologist. 2017;22(8):951-62.

35. Fernandes J, Bregman B, Combemale P, et al. Hospitalisation costs of metastatic melanoma in France; the MELISSA study (MELanoma In hoSpital coSts Assessment). BMC Health Serv Res. 2017;17(1):542. 